\title{
A closure model on velocity structure functions in homogeneous isotropic turbulence*
}

\author{
Le FANG ${ }^{1,2}$, Feng $\mathrm{GAO}^{3, \dagger}$ \\ 1. Laboratory of Mathematics and Physics, Ecole Centrale de Pékin, Beihang University, \\ Beijing 100191, China; \\ 2. Co-Innovation Center for Advanced Aero-Engine, Beihang University, Beijing 100191, China; \\ 3. Faculty of Engineering and Physical Sciences, University of Surrey, Guildford GU2 7XH, U. K.
}

\begin{abstract}
Closure models started from Chou's work have been developed for more than 70 years, aiming at providing analytical tools to describe turbulent flows in the spectral space. In this study, a preliminary attempt is presented to introduce a closure model in the physical space, using the velocity structure functions as key parameters. The present closure model appears to qualitatively reproduce the asymptotic scaling behaviors at small and large scales, despite some inappropriate behaviors such as oscillations. Therefore, further improvements of the present model are expected to provide appropriate descriptions of turbulent flows in the physical space.
\end{abstract}

Key words homogeneous isotropic turbulence, closure model, structure function

Chinese Library Classification $\mathrm{O} 357.5^{+} 1$

2010 Mathematics Subject Classification 76F55, 76F05

\section{Nomenclature}

$t, \quad$ time;

$\nu$ viscosity;

$r, \quad$ two-point distance;

$\alpha, \beta, \quad$ model coefficients;

$n$, scaling exponent;

$p, \quad$ scale-similarity exponent;

$c_{p}, \quad$ scale-similarity coefficient;

$D_{11}$, second-order longitudinal structure function;

\section{Introduction}

The closure modelling techniques are a fundamental theoretical tool for understanding and investigating the nonlinear behaviors of turbulent flows. The quasi-normal assumption, first introduced by $\mathrm{Chou}^{[1]}$, is the most famous and successful technique for obtaining closures in

* Received Oct. 22, 2016 / Revised May 12, 2017

Project supported by the National Natural Science Foundation of China (Nos. 11572025, 11202013, and 51420105008)

† Corresponding author, E-mail: f.gao@surrey.ac.uk 
the spectral space. Subsequent developments of the quasi-normal assumption led to the eddydamping quasi-normal Markovian (EDQNM) closure $^{[2-3]}$, which is usually regarded as one of the most accurate analytical models of turbulence. There are also some investigations on relaxing the quasi-normal assumption to consider the fourth-order accumulations ${ }^{[4-5]}$, and investigations on the possibility of using the restricted Euler (RE) assumption to replace the quasi-normal assumption ${ }^{[6]}$.

However, the quasi-normal assumption only provides a description in the spectral space which introduces links between second-order and fourth-order spectral correlations. This restricts its implementation in complex turbulent flows which are difficult to be described in the spectral space.

In the present study, we attempt to apply a closure to the fourth-order structure functions in the physical space, and discuss the possibility to reproduce the correct scaling behaviors. In particular, we discuss the pressure effects in the next-order structure function equations. The present approach is expected to inspire future studies on closure models in the physical space for describing complex turbulent flows.

\section{The model}

We consider a statistically stationary homogeneous isotropic turbulence field such that for any statistical quantity, there is $\partial_{t}\langle\cdot\rangle=0$, where $t$ is time, and \langle\rangle is ensemble average. The classical Kolmogorov equation can be simply written as ${ }^{[7-9]}$

$$
D_{111}=6 \nu D_{11}^{\prime}-\frac{4}{5} \epsilon r
$$

where $\nu$ is the kinematic viscosity, $r$ is the two-point distance, and $\epsilon$ is the dissipation rate. $D_{11}(r)=\left\langle\left(u_{1}\left(r e_{1}\right)-u_{1}(0)\right)^{2}\right\rangle$ and $D_{111}(r)=\left\langle\left(u_{1}\left(r e_{1}\right)-u_{1}(0)\right)^{3}\right\rangle$ are the second-order and thirdorder longitudinal velocity structure functions, respectively, with $e_{1}$ being the unit vector in the $x_{1}$-axis. Clearly, this equation shows the relation between the second-order and third-order structure functions.

The relation between the third-order and fourth-order structure functions can be found in Ref. [10]. Neglecting the non-stationary term $\partial_{t} D_{1111}$ and assuming the first-order Taylor approximation that $Z_{111} \approx 6 C$ in Ref. [10], we obtain

$$
D_{1111}^{\prime}+\frac{2}{r}\left(D_{1111}-3 D_{1122}\right)-10 \nu\left(\frac{4}{r^{2}} D_{111}-\frac{4}{r} D_{111}^{\prime}-D_{111}^{\prime \prime}\right)=-T_{111},
$$

where $D_{1111}(r)=\left\langle\left(u_{1}\left(r e_{1}\right)-u_{1}(0)\right)^{4}\right\rangle$ and $D_{1122}(r)=\left\langle\left(u_{1}\left(r e_{1}\right)-u_{1}(0)\right)^{2}\left(u_{2}\left(r e_{1}\right)-u_{2}(0)\right)^{2}\right\rangle$ are the fourth-order longitudinal and cross velocity structure functions, respectively, and $T_{111}(r)$ is a velocity-pressure correlation function.

In order to close the fourth-order structure functions in Eq. (2), we employ the extended scale similarity (ESS) theory ${ }^{[11-12]}$ which describes the relation between the fourth-order and second-order longitudinal structure functions, and the quasi-normal assumption which considers the relation between the fourth-order longitudinal and cross structure functions ${ }^{[6]}$.

The ESS theory implies that in a wide range, the fourth-order and second-order longitudinal structure functions can be expressed by a constant scale-similarity fractal scaling. From Table 2 of Ref. [12], this scaling can be estimated as $p=1.28 / 0.70 \approx 1.83$. McComb et al. ${ }^{[13]}$ showed that the ESS theory is valid at almost all scales in homogeneous isotropic turbulence (see Fig. 1 of Ref. [13]), which directly supports the present closure idea. Therefore, the following relation can be obtained:

$$
D_{1111}=c_{p} D_{11}^{p}
$$


where $c_{p}$ is a constant (see Subsection 4.1 for discussion).

Moreover, there are quite few studies on different fourth-order structure functions. Therefore, the quasi-normal assumption might be the only choice in the present model. From Eq. (3) in Ref. [6], by using the isotropy condition $D_{22}=D_{11}+\frac{r}{2} D_{11}^{\prime}$ and the scaling $D_{11}(r) \propto r^{2 / 3}$ in the inertial range (Subsection 4.3 for details), we can obtain

$$
D_{1122}=\frac{4}{9} D_{1111}=\frac{4}{9} c_{p} D_{11}^{p} .
$$

Although we have not found any direct support for this relation, there is numerical evidence that implies the rationality of using the quasi-normal assumption to consider the relation between the fourth-order longitudinal and cross structure functions. An example was given in Fig. 4(a) of Ref. [6]. The quasi-normal (i.e., the "Gaussian" state) approximation works better than the RE approximation in many situations in equilibrium turbulence and low Mach turbulence. However, in order to consider the non-equilibrium procedures, one needs to consider the Gaussian-RE line ${ }^{[6]}$ instead of the quasi-normal assumption in Eq. (4). The Gaussian-RE line involves an additional parameter on the non-equilibrium property ${ }^{[14]}$. In the present paper, we only focus on the statistically stationary turbulence where the flow cannot be non-equilibrium. Thus, the quasi-normal assumption is acceptable. Actually, as will be discussed in Subsection 4.3, different formulations for the cross structure function do not qualitatively affect the results.

From Eqs. (1)-(4), we can finally obtain the following equation:

$$
p c_{p} r^{2} D_{11}^{p-1} D_{11}^{\prime}-\frac{2}{3} c_{p} r D_{11}^{p}-240 \nu^{2} D_{11}^{\prime}+240 \nu^{2} r D_{11}^{\prime \prime}+60 \nu^{2} r^{2} D_{11}^{\prime \prime \prime}=-r^{2} T_{111} .
$$

This equation keeps only the second-order longitudinal velocity structure function rather than the higher-order moments. However, there is still a velocity-pressure correlation term $-T_{111}$ on the right-hand side which cannot be simply neglected. There is currently no convincing theory for closing this terms, while existing models often require complicated phenomenological assumptions ${ }^{[15-17]}$. In order to simplify this model, we note the observations from Figs. 1 and 2 of Ref. [10] that $-T_{111}$ is approximately proportional to both $D_{1111}^{\prime}+\frac{2}{r} D_{1111}$ and $\frac{6}{r} D_{1122}$ at all scales. Therefore, by using the quasi-normal assumption in Eq. (4) and the isotropic condition, we assume either a proportional relation

$$
-T_{111}=\alpha\left(D_{1111}^{\prime}+\frac{2}{r} D_{1111}\right)=\alpha\left(p c_{p} D_{11}^{p-1} D_{11}^{\prime}+\frac{2 c_{p}}{r} D_{11}^{p}\right)
$$

with $\alpha$ being a model constant, or a proportional relation

$$
-T_{111}=\beta\left(\frac{6}{r} D_{1122}\right)=\beta\left(\frac{8 c_{p}}{3 r} D_{11}^{p}\right)
$$

with $\beta$ being another model constant.

We will prove that from a scaling law, the values of $\alpha$ and $\beta$ can be analytically obtained. Suppose the classical $2 / 3$ scaling in the inertial range, i.e., $D_{11}(r) \propto r^{2 / 3}$. From Eqs. (5) and (7), we can finally obtain

$$
(3 p-3-3 p \alpha-9 \alpha) c_{p} r^{(4 / 3+2 p / 3)}-880 \nu^{2}=0,
$$

which yields $\alpha=(p-1) /(p+3)$ for large $r$ (corresponding to the inertial scales). A value of $\alpha=0.1718$ can be derived using the value of $p=1.83$ obtained previously by the ESS theory. Similarly, from Eqs. (5) and (7), we can finally obtain

$$
(3 p-3-12 \beta) r^{(4 / 3+2 p / 3)}-880 \nu^{2}=0,
$$


which yields $\beta=(p-1) / 4$ for large $r$. The model constant of $\beta=0.2075$ is calculated with the same value of $p=1.83$ by the ESS theory.

Combining Eqs. (6) and (5) leads to the $\alpha$ formulation,

$$
(1-\alpha) p c_{p} r^{2} D_{11}^{p-1} D_{11}^{\prime}-\left(\frac{2}{3}+2 \alpha\right) c_{p} r D_{11}^{p}-240 \nu^{2} D_{11}^{\prime}+240 \nu^{2} r D_{11}^{\prime \prime}+60 \nu^{2} r^{2} D_{11}^{\prime \prime \prime}=0,
$$

while substituting Eq. (7) into Eq. (5) leads to the $\beta$ formulation,

$$
p c_{p} r^{2} D_{11}^{p-1} D_{11}^{\prime}-\left(\frac{2}{3}+\frac{8 \beta}{3}\right) c_{p} r D_{11}^{p}-240 \nu^{2} D_{11}^{\prime}+240 \nu^{2} r D_{11}^{\prime \prime}+60 \nu^{2} r^{2} D_{11}^{\prime \prime \prime}=0 .
$$

Equation (10) or (11) then defines a closure model on velocity structure functions in isotropic turbulence.

Similarly, if we consider the anomalous scaling laws ${ }^{[18]}$ instead of the $2 / 3$ scaling in the inertial range, the values of $\alpha$ and $\beta$ can also be calculated. For example, supposing $D_{11} \propto r^{0.7}$ will yield the constants $\alpha=(0.7 p-2 / 3) /(0.7 p+2)$ and $\beta=3(0.7 p-2 / 3) / 8$. When $p=1.83$, they are, respectively, $\alpha=0.1872$ and $\beta=0.2304$.

\section{Results}

Both Eqs. (10) and (11) are nonlinear. Thus, it is difficult to find analytical general solutions to them. Instead, we present the corresponding numerical solutions in this section to validate these models. Some typical parameters are defined as initial conditions such as

$$
D_{11}(0.01)=0.0001, \quad D_{11}^{\prime}(0.01)=0.02, \quad D_{11}^{\prime \prime}(0.01)=2 .
$$

Here, the value of $D_{11}(0.01)$ is arbitrarily defined, while the values of $D_{11}^{\prime}(0.01)$ and $D_{11}^{\prime \prime}(0.01)$ are defined to guarantee that $D_{11}(r) \propto r^{2}$ at very small $r$. The $\alpha$ formulation and $\beta$ formulation are tested independently. We also perform two groups of simulations to compare the results obtained by different scaling laws for $D_{11}$. The parameters $c_{p}=0.01$ and $\nu=0.1$ are artificially defined. Results are shown in Fig. 1, where the scaling exponent is calculated as $n(r)=$ $r D_{11}^{\prime} / D_{11}^{[9]}$. Clearly, in each case, the scaling exponent is 2 at small scales and approaches the expected scaling (i.e., $2 / 3$ and 0.7 , respectively) at large scales. This behavior is qualitatively in agreement with the literature ${ }^{[9,19]}$, but in the present study, all curves show oscillations, which contradict the monotonous values in the literature. These oscillations are perhaps caused by the inappropriate assumptions on the pressure term, and will be discussed in Subsection 4.2. Also, in each group, the $\alpha$ and $\beta$ formulations yield similar results, illustrating the consistency between the two formulations.

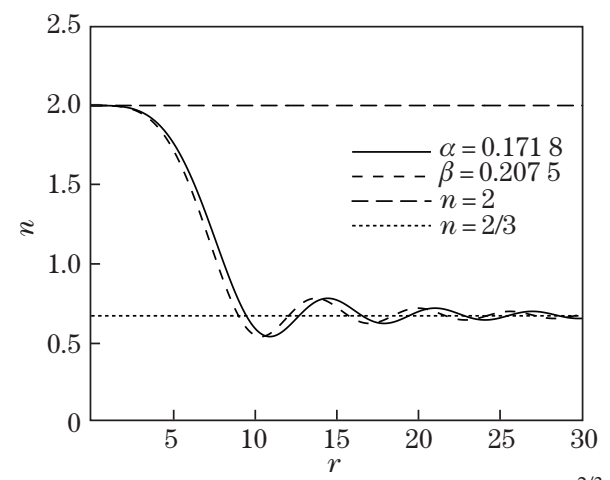

(a) Using the classical Kolmogorov scaling $r^{2 / 3}$

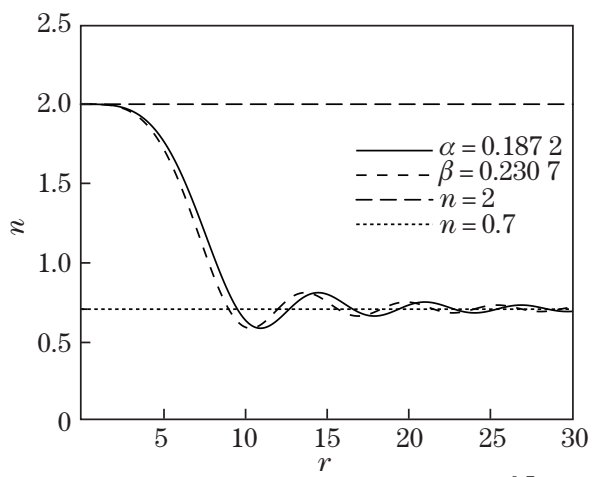

(b) Using the anomalous scaling $r^{0.7}$

Fig. 1 Scaling exponent $n$ calculated with the closure model where $c_{p}=0.01$, and $\nu=0.1$. The $\alpha$ formulation and $\beta$ formulation are employed, respectively 


\section{Discussion}

\subsection{Model parameters}

In the previous section, we have shown that the $\alpha$ formulation and $\beta$ formulation produce similar results, while the values of $\alpha$ and $\beta$ are directly related to the asymptotic scaling exponent in the inertial range. In this section, the sensitivities to other model parameters, such as the ESS coefficient $c_{p}$ and the viscosity $\nu$, will be discussed. For brevity, we will use the $\alpha$ formulation with the classical Kolmogorov scaling $r^{2 / 3}$ in the following discussion.

From the ESS theory, the self similarity between structure functions is fractal (i.e., $p=1.83$ instead of $p=2$ ). We emphasize that this does not mean that the Galilean invariance is broken, since all phenomena in the ESS theory must be observed by using the root-mean-square velocity $u^{\prime}$ for nondimensionalization. This implies that although formally $c_{p}$ is fractal, the turbulence dissipative scale is always considered to determine the coefficient $c_{p}$. The sensitivity of the model to different values of $c_{p}$ is shown in Fig. 2. Clearly, when we replace the original length scale $r$ with the fractal length scale $c_{p}^{\frac{1}{2 p}} r$, the scaling exponents are in excellent coincidence with each other. Because in Eqs. (10) and (11), the first two terms (which are dominant for large $r$ ) have the spatial dimension $c_{p} r^{2 p+1}$, replacing $r$ with $c_{p}^{\frac{1}{2 p}} r$ leads to the vanishing of $c_{p}$. In brief, the ESS coefficient $c_{p}$ represents a spatial scaling with consideration of the turbulence dissipative scale.

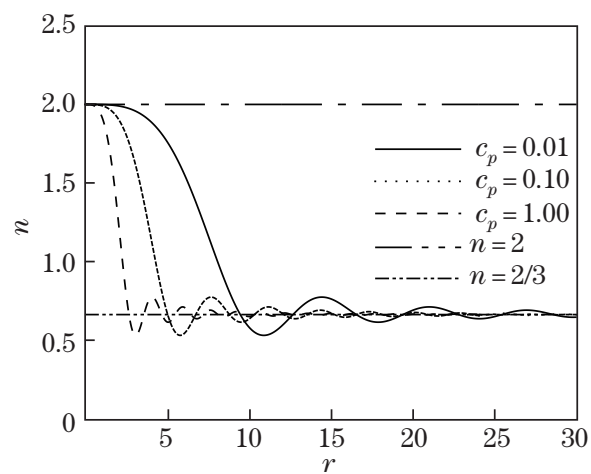

(a) Using the original length scale $r$

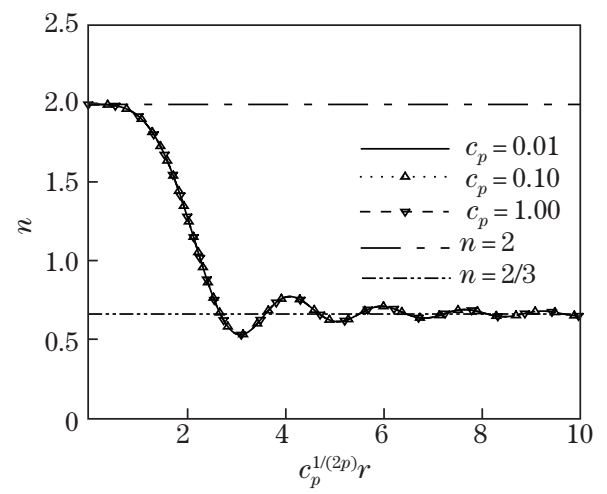

(b) Using the fractal length scale $c_{p}^{1 /(2 p)} r$

Fig. 2 Scaling exponent $n$ calculated with the closure model with different values of $c_{p}$. The $\alpha$ formulation is employed by using the classical Kolmogorov scaling $r^{2 / 3}$. Viscosity $\nu=0.1$

The role of $\nu$ in the present model is similar to that of $c_{p}$, which corresponds to a spatial scaling. From Eqs. (10) and (11), it is clear that $c_{p} \sim \nu^{-2}$. Hence, in Fig. 3, we can use the length scale $\nu^{-1 / p} r$ to achieve coincidences among different cases.

\subsection{Corrections to the model of $T_{111}$}

As discussed in the previous section, the oscillating phenomenon is unphysical. This may be caused by the inappropriate assumption on the pressure term $T_{111}$. In Section 2 , we assumed that the coefficients $\alpha$ and $\beta$ are constant, which are determined by an asymptotic analysis for large $r$. However, for small $r$, there should be another asymptotic limit and thus a transition against $r$ for the coefficients $\alpha$ and $\beta$. When $r$ is small, from the Taylor expansion, we have $D_{11}(r) \propto r^{2}$. Then, from Eqs. (5) and (6), we can obtain

$$
\alpha_{s}=\frac{3 p-2}{3 p+6}, \quad \beta_{s}=\frac{3 p-2}{8},
$$




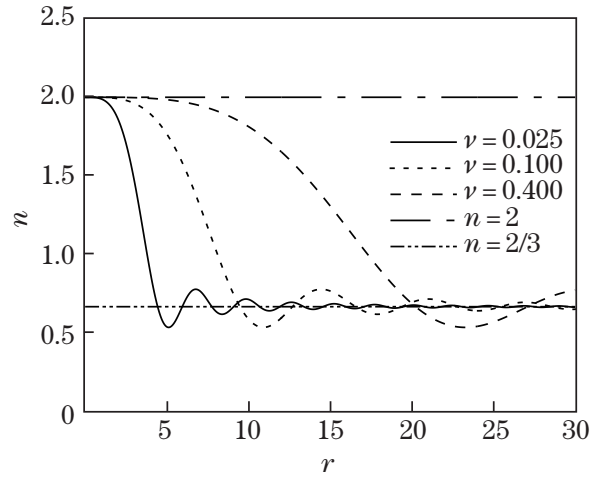

(a) Using the original length scale $r$

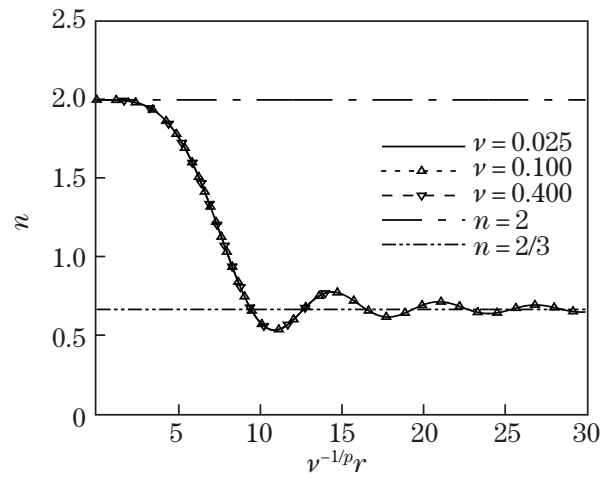

(b) Using the feactal length scale $v^{-1 / p} r$

Fig. 3 Scaling exponent $n$ calculated with the closure model with different values of $\nu$. The $\alpha$ formulation is employed by using classical Kolmogorov scaling $r^{2 / 3}$. ESS coefficient $c_{p}=0.01$

which differs from the inertial-scale results $\alpha_{l}=(p-1) /(p+3)$ and $\beta_{l}=(p-1) / 4$. In order to introduce a transition between these values, we introduce an exponential function, and the transitional $\alpha(r)$ writes

$$
\alpha(r)=\alpha_{l}+\left(\alpha_{s}-\alpha_{l}\right) \exp \left(-c_{1} r\right)
$$

with $c_{1}$ being constant. The results with $c_{1}=0.1$ are plotted in Fig. 4 in comparison with the constant $\alpha$ formulation. A better asymptotic behavior is observed with a reduced oscillating amplitude. This suggests the possibility of using a transition model in the closure model, and that the present closure model has potential to be improved by using an appropriate approximation for the pressure term $T_{111}$.

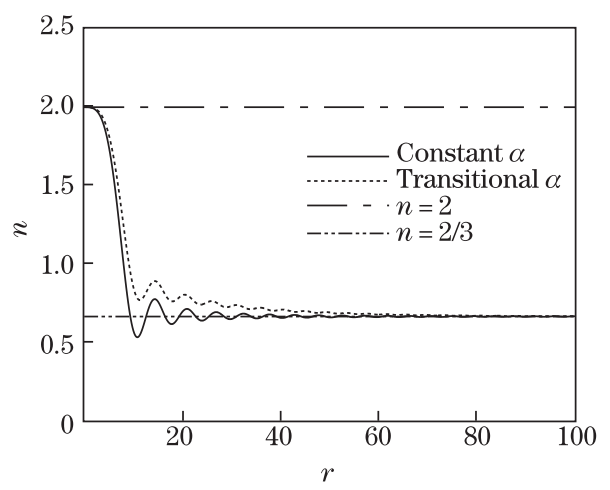

Fig. 4 Scaling exponent $n$ calculated with the closure model with different $\alpha$ formulations, where $c_{p}=0.01, \nu=0.1$, and $c_{1}=0.1$

\subsection{On the closure of the fourth-order cross structure function}

In the present closure model, we use the quasi-normal assumption to close the fourth-order cross structure function. Here, we present more details about this derivation and discuss the role of the Gaussian-RE line.

In the quasi-normal assumption, there are relations,

$$
D_{1111}=3 D_{11}^{2}, \quad D_{1111}=D_{11} D_{22} .
$$

Equation (4) can be derived in the inertial range by applying the classical scaling $r^{2 / 3}$ under the isotropy condition $D_{22}=D_{11}+\frac{r}{2} D_{11}^{\prime}$. However, this equation is not true in the dissipative 
range where $D_{11} \propto r^{2}$ and $D_{22}=2 D_{11}{ }^{[20]}$, and there will be ${ }^{[6]}$

$$
D_{1122}=\frac{2}{3} D_{1111}
$$

Moreover, we can also use the RE assumption to close the fourth-order cross structure function. The RE assumption yields ${ }^{[6]}$

$$
D_{1111}^{\prime}+\frac{2}{r} D_{1111}-\frac{6}{r} D_{1122}=0
$$

In the inertial range, if we employ the scaling law $D_{1111} \propto r^{q}$ with $q \approx 1.28^{[18]}$, there is

$$
D_{1122}=\frac{2+q}{6} D_{1111}
$$

In the dissipative range, the Taylor expansion $D_{1111} \propto r^{4}$ leads to

$$
D_{1122}=D_{1111}
$$

Therefore, there are four different relations between $D_{1111}$ and $D_{1122}$, i.e., Eqs. (4), (16), (18), and (19), derived under different assumptions and scales, respectively. All these formulations with various related transitional models are tested, but the unphysical oscillations are not eliminated (for brevity, these results are not plotted). Therefore, we remark that the relation between $D_{1111}$ and $D_{1122}$ is not a dominant factor in the present closure.

\section{Conclusions}

The closure of turbulence field is a longstanding fundamental problem, while most closure models are introduced in the spectral space. In this paper, we present a preliminary attempt to close the fourth-order structure functions via the second-order longitudinal structure function. In this model, the Kolmogorov equation and the next-order equation by Hill and Boratav are used as the basic equations, while both the ESS theory and the quasi-normal assumption for fourth-order moments are employed. In addition, a linear model for the pressure-velocity correlation term is introduced.

The present closure model successfully reproduces the asymptotic scalings, which can be classical $r^{2 / 3}$ or anomalous $r^{0.7}$, for both small and large scales in statistically stationary homogeneous isotropic turbulence. Both the $\alpha$ and $\beta$ formulations lead to similar results. Due to the fractal formulations, the parameters $c_{p}$ and $\nu$ are normalized by the root-mean square velocity $u^{\prime}$, implying the relative scales to the dissipative scale. The distance $r$ can be re-scaled by $c_{p}^{\frac{1}{2 p}} \nu^{-\frac{1}{p}} r$.

The oscillations in the results are unphysical and may be caused by the inappropriate assumption of the pressure-velocity correlation. A transition $\alpha$ formulation with artificial interpolation is shown to improve the results, suggesting that the pressure models have potential to be improved by an appropriate consideration of the pressure-velocity correlations.

Being different from the existing closures in the spectral space, the proposed model has the potential to be employed in complex flows in the physical space. Further improvements of this model are expected to take into account the mean velocity or non-stationarity with appropriate relaxation of the homogeneity condition. Also, in addition to the longitudinal components considered in the present paper, the summation of $D_{i i}$ which describes anisotropic complex flows could be another possibility. 


\section{References}

[1] Chou, P. Y. On velocity correlations and the solution of equation of turbulent fluctuations. Quaterly Applied Mathematics, 3, 38-54 (1945)

[2] Lesieur, M. Turbulence in Fluids, Kluwer Academic, Dordrecht (1997)

[3] Orszag, S. A. Lectures on the statistical theory of turbulence. Fluid Dynamics, Les Houches Summer School of Theoretical Physics (eds. Balian, R. and Peube, J. L.), Gorden and Breach, New York (1974)

[4] Bos, W. J. T. and Rubinstein, R. On the strength of the nonlinearity in isotropic turbulence. Journal of Fluid Mechanics, 733, 158-170 (2013)

[5] Bos, W., Rubinstein, R., and Fang, L. Reduction of mean-square advection in turbulent passive scalar mixing. Physics of Fluids, 24(7), 075104 (2012)

[6] Fang, L., Zhang, Y. J., Fang, J., and Zhu, Y. Relation of the fourth-order statistical invariants of velocity gradient tensor in isotropic turbulence. Physical Review E, 94(2), 023114 (2016)

[7] Fang, L. Applying the Kolmogorov Equation to the Problem of Subgrid Modeling for Large-Eddy Simulation of Turbulence, Ph. D. dissertation, Ecole centrale de Lyon (2009)

[8] Tatarskii, V. I. Use of the 4/5 Kolmogorov equation for describing some characteristics of fully developed turbulence. Physics of Fluids, 17, 035110 (2005)

[9] Fang, L., Bos, W. J. T., Zhou, X. Z., Shao, L., and Bertoglio, J. P. Corrections to the scaling of the second-order structure function in isotropic turbulence. Acta Mechanica Sinica, 26(2), 151-157 (2010)

[10] Hill, R. J. and Boratav, O. N. Next-order structure-function equations. Physics of Fluids, 13, 276-283 (2001)

[11] Benzi, R., Ciliberto, S., Tripiccione, R., Baudet, C., Massaioli, F., and Succi, S. Extended scalesimilarity in turbulent flows. Physical Review E, 48(1), R29-R32 (1993)

[12] Benzi, R., Ciliberto, S., Baudet, C., and Chavarria, G. R. On the scaling of three-dimensional homogeneous and isotropic turbulence. Physica D, 80, 385-398 (1995)

[13] McComb, W. D., Yoffe, S. R., Linkmann, M. F., and Berera, A. Spectral analysis of structure functions and their scaling exponents in forced isotropic turbulence. Physics of Fluids, 90, 053010 (2014)

[14] Fang, L., Zhu, Y., Liu, Y. W., and Lu, L. P. Spectral non-equilibrium property in homogeneous isotropic turbulence and its implication in subgrid-scale modeling. Physics Letters A, 379(38), 2331-2336 (2015)

[15] Chevillard, L., Meneveau, C., Biferale, L., and Toschi, F. Modeling the pressure Hessian and viscous Laplacian in turbulence: comparisons with direct numerical simulation and implications on velocity gradient dynamics. Physics of Fluids, 20, 101504 (2008)

[16] Chevillard, L. and Meneveau, C. Lagrangian dynamics and statistical geometric structure of turbulence. Physics Review Letters, 97, 174501 (2006)

[17] Wilczek, M. and Meneveau, C. Pressure Hessian and viscous contributions to velocity gradient statistics based on Gaussian random fields. Journal of Fluid Mechanics, 756, 191-225 (2014)

[18] She, Z. S. and Leveque, E. Universal scaling law in fully developed turbulence. Physics Review Letters, 72, 336-339 (1994)

[19] Bos, W. J. T., Chevillard, L., Scott, J., and Rubinstein, R. Reynolds number effects on the velocity increment skewness in isotropic turbulence. Physics of Fluids, 24, 015108 (2012)

[20] Batchelor, G. K. The Theory of Homogeneous Turbulence, Cambridge University Press, Cambridge (1953) 